\title{
Single-mode output by controlling the spatiotemporal nonlinearities in mode-locked femtosecond multimode fiber lasers
}

\author{
Uğur Teğin $\odot,{ }^{\text {a,b,* }}$ Babak Rahmani, ${ }^{b}$ Eirini Kakkava $\odot,{ }^{a}{ }^{a}$ Demetri Psaltis, ${ }^{a}$ and Christophe Moser ${ }^{b}$ \\ áÉcole Polytechnique Fédérale de Lausanne, Optics Laboratory, Lausanne, Switzerland \\ 'École Polytechnique Fédérale de Lausanne, Laboratory of Applied Photonics Devices, Lausanne, Switzerland
}

\begin{abstract}
The performance of fiber mode-locked lasers is limited due to the high nonlinearity induced by the spatial confinement of the single-mode fiber core. To massively increase the pulse energy of the femtosecond pulses, amplification is performed outside the oscillator. Recently, spatiotemporal mode-locking has been proposed as a new path to fiber lasers. However, the beam quality was highly multimode, and the calculated threshold pulse energy (>100 nJ) for nonlinear beam self-cleaning was challenging to realize. We present an approach to reach high energy per pulse directly in the mode-locked multimode fiber oscillator with a near single-mode output beam. Our approach relies on spatial beam self-cleaning via the nonlinear Kerr effect, and we demonstrate a multimode fiber oscillator with $M^{2}<1.13$ beam profile, up to $24 \mathrm{~nJ}$ energy, and sub-100 fs compressed duration. Nonlinear beam self-cleaning is verified both numerically and experimentally for the first time in a mode-locked multimode laser cavity. The reported approach is further power scalable with larger core sized fibers up to a certain level of modal dispersion and could benefit applications that require high-power ultrashort lasers with commercially available optical fibers.
\end{abstract}

Keywords: fiber lasers; spatiotemporally mode-locked lasers; multimode nonlinear fiber optics.

Received Jul. 17, 2020; revised manuscript received Sep. 1, 2020; accepted for publication Sep. 17, 2020; published online Oct. 16, 2020.

(C) The Authors. Published by SPIE and CLP under a Creative Commons Attribution 4.0 Unported License. Distribution or reproduction of this work in whole or in part requires full attribution of the original publication, including its DOI.

[DOI: $10.1117 / 1 . A P .2 .5 .056005]$

\section{Introduction}

Fiber laser dynamics have been studied extensively in the past decades to generate femtosecond pulses with high energies and peak powers. ${ }^{1}$ Numerous laser designs are developed to understand nonlinear wave propagation under partial feedback conditions. By tuning complex cavity dynamics in single-mode fiber cavities, self-organization of longitudinal cavity modes with various temporal profiles and central wavelengths has been realized, such as soliton, ${ }^{2}$ similariton, ${ }^{3,4}$ and dissipative soliton. ${ }^{5,6}$ Due to the high spatial confinement in the small single-mode fiber core, nonlinear effects appear at moderate peak power in solid cores, and the accumulation of excessive nonlinear phase leads to pulse breakup, which then limits the achievable pulse energies.

To overcome this limitation, custom-made sophisticated fibers, having large single-mode areas in a photonic crystal fiber,

*Address all correspondence to Uğur Teğin, E-mail: ugur.tegin@epfl.ch were proposed to reach the microjoule pulse energy level. ${ }^{7,8}$ The fiber used in these demonstrations with its mode field diameter of $70 \mu \mathrm{m}$ needed to be kept straight to avoid bending losses and ensure stability. They share the same limitations as solid-state lasers in the sense that they are rigid and cannot be spliced with conventional techniques. As a result, such lasers do not share the features of fiber lasers that render them advantageous in practice.

Recently, spatiotemporal mode-locking has been demonstrated in commercially available multimode fiber (MMF) cavities with graded-index multimode fibers (GRIN MMFs) by harnessing their low modal dispersion and inherent periodic self-focusing to produce a coherent superposition of transverse and longitudinal modes in an all-normal dispersion regime. ${ }^{9-11}$ These studies presented cavities with dissipative soliton pulse operation with low-output beam quality despite utilizing gain fibers with three modes. In a recent study, improvement in the output beam profile of a spatiotemporal mode-locked fiber laser 
was reported by changing the generated pulse type from a dissipative soliton to an amplifier similariton. The reported pulses lead to pulse energies of $2.4 \mathrm{~nJ}$ with output beam $M^{2}$ value $<1.4$. $^{12}$ Although a better beam profile was reported compared to previous results, the single-mode beam profile $\left(M^{2} \sim 1\right)$ could not be achieved due to the limitations of amplifier similariton pulse type such as the degradation of pulse quality and peak power with increasing pulse energy.

In this paper, we demonstrate an approach to generate $>20 \mathrm{~nJ}$, sub-100 fs pulses with near Gaussian output beam shape by controlling the spatiotemporal cavity dynamics of multimode fiber lasers. This pulse energy represents a tenfold improvement over previously reported MMF oscillators with a Gaussian beam shape. Moreover, our method is limited only by the damage of the threshold fiber splices and can be increased to the microjoule energy level. The key element in the novel design that enables the increased pulse energy is the Kerrinduced beam self-cleaning in a GRIN MMF ${ }^{13}$ that occurs when a high-intensity pulse propagates in the fiber.

The optical wave propagation inside the cavity is designed to synergistically achieve nonlinear beam cleaning and spatiotemporal mode-locking. With our approach, spatiotemporally mode-locked fiber lasers overcome the power limitations of mode-locked single-mode fiber lasers without sacrificing the output beam quality. Moreover, the presented approach is not limited to the demonstrated power levels and theoretically scalable with standard large core multimode fibers up to a certain level of modal dispersion.

We experimentally demonstrate that the highly multimode beam profile observed at the output of a continuous wave multimode fiber cavity is transformed to a stable Gaussian beam profile when the oscillator is spatiotemporally mode-locked. Our numerical studies verified that there is an energy exchange from higher-order modes to lower order modes in the propagating GRIN MMF section of the laser cavity for the experimentally achieved power level. Inside the spatiotemporal mode-locked cavity, Kerr-induced beam self-cleaning creates a minimum loss condition to the emerging mode-locked pulses. The reported multimode fiber laser generates sub-100 fs pulses with high pulse energy (>20 nJ) and good beam quality if the $M^{2}$ value is $<1.13$.

\section{Methods}

\subsection{Multimode Oscillator Simulations}

Numerical simulations for mode-locked pulse formation are conducted for the model used by Tegin et al. ${ }^{12}$ For GRIN MMF sections of the cavity, a multimode nonlinear Schrödinger equation ${ }^{14}$ is solved by considering the five linearly polarized modes (more details of simulations can be found in Supplementary Discussion I in the Supplementary Material). Simplifications such as simulating few-mode fiber sections as single mode to decrease the computation time and defining coupling ratios before and after the GRIN MMF sections to mimic the effect of splice points are performed. Simulations are initiated with quantum noise fields. The integration step for GRIN MMF sections is defined as the ratio of the self-imaging period of the fiber with four. For the simulation result shown in Fig. 1, the gain fiber is modeled with a Lorentzian gain shape with $50 \mathrm{~nm}$ bandwidth, $30 \mathrm{~dB}$ small-signal gain, and $3.2 \mathrm{~nJ}$ saturation energy. The coupling condition between the few-mode gain fiber and GRIN MMF is simulated as [20\%, 30\%, 20\%, 20\%, and $10 \%$ ]. The intracavity spatial filtering is applied to the

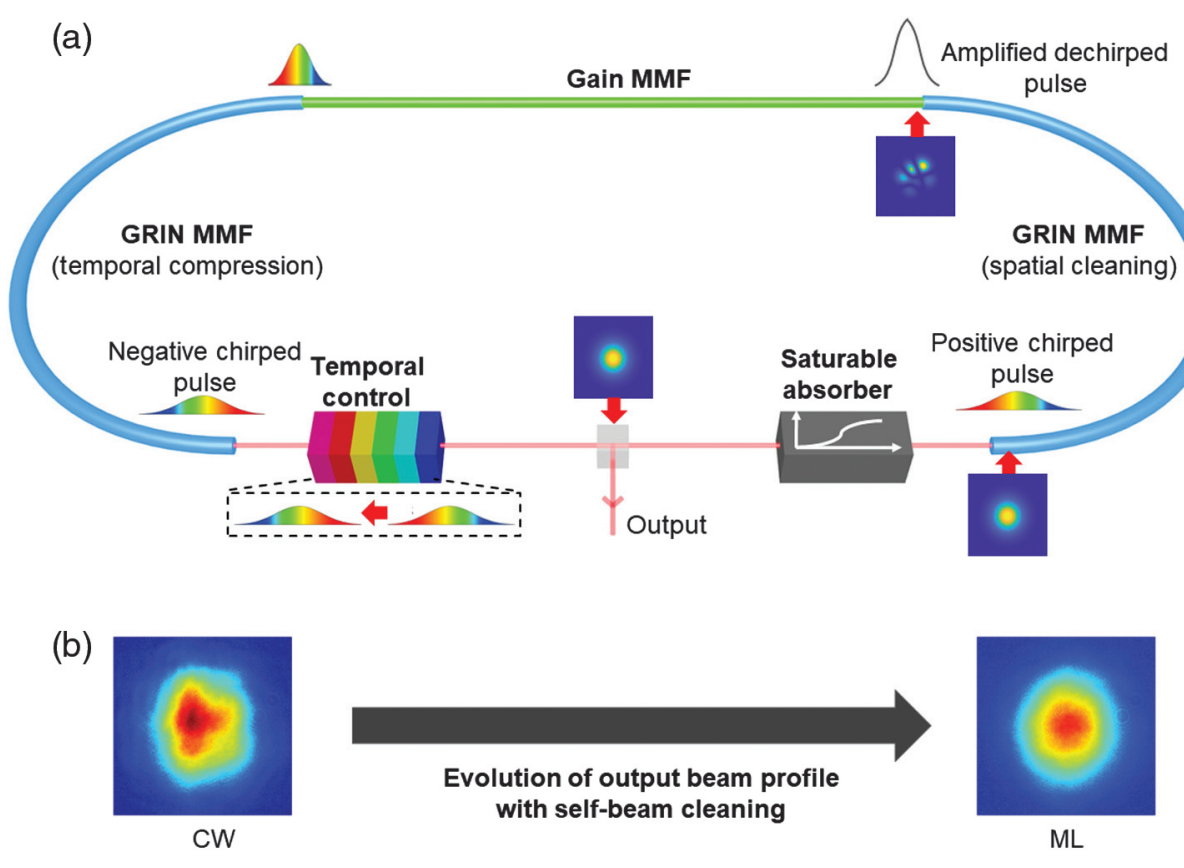

Fig. 1 Conceptual outline of the multimode fiber cavity and schematic of spatiotemporal modelocking with beam self-cleaning. (a) Conceptual outline of dispersion-managed cavity design with indicated temporal dynamics. Laser pumping scheme is not presented. (b) Schematic of modelocking mechanism and experimentally measured output beam profile evolution. $\mathrm{CW}$, continuous wave; ML, mode-locked. 
considered modes by allowing their transmission with [50\%, $50 \%, 50 \%, 50 \%$, and $0 \%$ ]. The coupling for the field propagating from the GRIN MMF to a few-mode fiber is calculated as a summation of modes with the transmission coefficients $[100 \%$, $100 \%, 100 \%, 0 \%$, and $0 \%]$.

\subsection{Single-Pass Beam Cleaning Simulations}

The simulations regarding beam cleaning are performed by numerically solving a multimode nonlinear Schrödinger equation with high numerical accuracy and small integration step. To achieve this goal, the integration step is defined as the ratio of the self-imaging period of GRIN MMF with 20, and the fourthorder Runge-Kutta in the Interaction Picture method is used for accuracy. ${ }^{15}$ (Further details can be found in Supplementary Discussion I in the Supplementary Material.)

\subsection{Experiments}

A $20 \mathrm{~W}, 976 \mathrm{~nm}$ pump diode (II-VI Photonics BMU20-976S01-R) is spliced to the oscillator cavity with a pump combiner [Lightel MPC $(2+1) \times 1$ ] compatible with a $1.3 \mathrm{~m}$ MMF gain section (nLight Yb-1200 10/125). To excite the higher-order modes of the GRIN MMF (Thorlabs GIF50E), the gain fiber is spliced to the $1.4 \mathrm{~m}$ GRIN MMF with a small offset (5 $\mu \mathrm{m})$ and coiled with a $30 \mathrm{~cm}$ diameter to encourage multimode pulse propagation. After the first GRIN MMF section, light is collimated and travels through the wave plates, polarizing beam splitter, isolator, grating pair (600 lines/mm), and spatial filter (randomly placed pinhole). Spatial filtering in spatiotemporal mode-locking is one of the key elements. Its purpose is to restrict the modal content of the intracavity beam. In our experiments with multimode laser cavities, we observed that, in the absence of modal filtering, the mode-locking experiences oscillations in long-term operations. In the presented cavity, the effect of the randomly placed pinhole produces experimental mode-locking stability, and, for different positioning of the pinhole, the spatial beam profiles remained the same. With wave plates and a polarizing beam splitter, nonlinear polarization evolution (NPE) is implemented as an artificial saturable absorber. The additional half-wave plate is placed between the polarization-dependent isolator and the grating pair to control the first-order reflection efficiency of the gratings. The free-space ends of the GRIN MMFs are angle-cleaved to eliminate parasitic back reflections. Another GRIN MMF section with $1 \mathrm{~m}$ length establishes the ring cavity loop. Self-starting modelocking was achieved by adjusting the intracavity wave plates (see Video S1 [URL: https://doi.org/10.1117/1.AP.2.5.056005.1]). Spectrum measurements are performed with an optical spectrum analyzer (Ando AQ6317B) and spectrometer (Ocean Optics HR4000-CG-UV-NIR). Beam profile measurements are performed with CMOS cameras (Edmund Optics EO-32121M and Thorlabs DCC1545M). Temporal pulse measurements are performed with an autocorrelator (Femtochrome FR-103). The radio frequency spectrum is measured with an HP 3585A spectrum analyzer, and $>60 \mathrm{~dB}$ sideband suppression is reported (Fig. S10 in the Supplementary Material).

\section{Numerical Studies}

Our approach is inspired by dispersion-managed mode-locking in single-mode lasers, which is a method that was initially proposed to overcome the power limitation of soliton mode-locked lasers. ${ }^{16,17}$ Inside a dispersion-managed cavity, the pulse stretches and compresses significantly every round trip while having a near zero net-cavity group-velocity dispersion (GVD). This approach leads to the generation of ultrashort pulses (sub-100 fs) with broad-spectrum and high energy per pulse. ${ }^{18,19}$

In our study, the dispersion-managing approach is adapted to a multimode fiber cavity with a similar motivation, to generate intracavity short pulses with high peak powers sufficient to trigger strong spatiotemporal interactions in order to effect spatial beam clean-up. The proposed cavity is illustrated in Fig. 1(a). Silica fibers feature positive GVD for the emission wavelengths of the $\mathrm{Yb}$ fiber and stretch the propagating pulse with positive chirp. In our design, a grating pair is placed as a dispersionbalancing section to provide negative GVD values and change the sign of the chirp on the pulse. Such a temporal change causes compression of the propagating pulses in the following fiber sections (GRIN MMF and gain MMF).

We used the multimode nonlinear Schrödinger equation to numerically simulate the multimode laser to analyze pulse propagation dynamics and determine cavity parameters. A stable mode-lock regime after a few round-trips around net cavity GVD $\beta_{(2) \text { net }}=0.013 \mathrm{ps}^{2}$ is achieved, and dispersion-managed soliton pulse formation is numerically obtained with various powers and cavity excitation conditions. An example of the evolution of the intracavity pulse in one round trip is illustrated in Fig. 2 as a function of position inside the multimode cavity. By engineering the cavity dynamics, the shortest pulse duration is achieved at the end of the gain fiber section, where the amplification is the maximum, such that the highest intracavity peak power is obtained in the GRIN MMF section. This unique design can allow pulses to reach the Kerr-induced beam cleaning threshold inside the cavity. In our simulations, the pulse experiences more than 6 times spectral broadening in one round-trip. The temporal profile and spectrum of the output pulse measured at the NPE port are presented in Figs. S1a and S1b in the Supplementary Material. For the simulated cavity, mode-locked pulses with $5.1 \mathrm{~nJ}$ energy and 2.12 ps duration Gaussian-like temporal shape are generated from quantum noise. The numerically obtained output beam profile with the considered modes is presented in Fig. S1c in the Supplementary Material. For further details and simulations with different multimode fiber excitation conditions, see Sec. 2 and also Supplementary Discussion I in the Supplementary Material.

To investigate the possibility of the Kerr-induced beam selfcleaning in the cavity, single-pass numerical simulations were performed with high numerical accuracy. The energy exchange behavior between the modes is presented in Fig. 3. The modelocking simulations demonstrated in Fig. 2 suggest $\sim 450$ fs pulse duration after the gain section, and we numerically investigate the effect of intracavity pulse energy on energy exchange between the modes of GRIN MMF with different initial excitation scenarios. For the excitation case applied in mode-locking simulations between the simulated modes, when the pulse energy reaches $20 \mathrm{~nJ}$, energy fluctuations between the simulated modes start to decrease. For a $30 \mathrm{~nJ}$ pulse, the fundamental mode starts to increase its energy content, and, for $40 \mathrm{~nJ}$ pulse, it becomes dominant at the end of the GRIN MMF section of the cavity [see Fig. 3(a)]. For different excitation cases, the required pulse energy to achieve similar modal interaction is observed to be lower, as is shown in Figs. 3(b) and 3(c). For some cases, we observed that instead of the fundamental mode, an alternative low-order mode such as $\mathrm{LP}_{11 \mathrm{~b}}$ is observed as a leading mode at the end of GRIN MMF for $20 \mathrm{~nJ}$ pulse energy [see Fig. 3(d)]. 


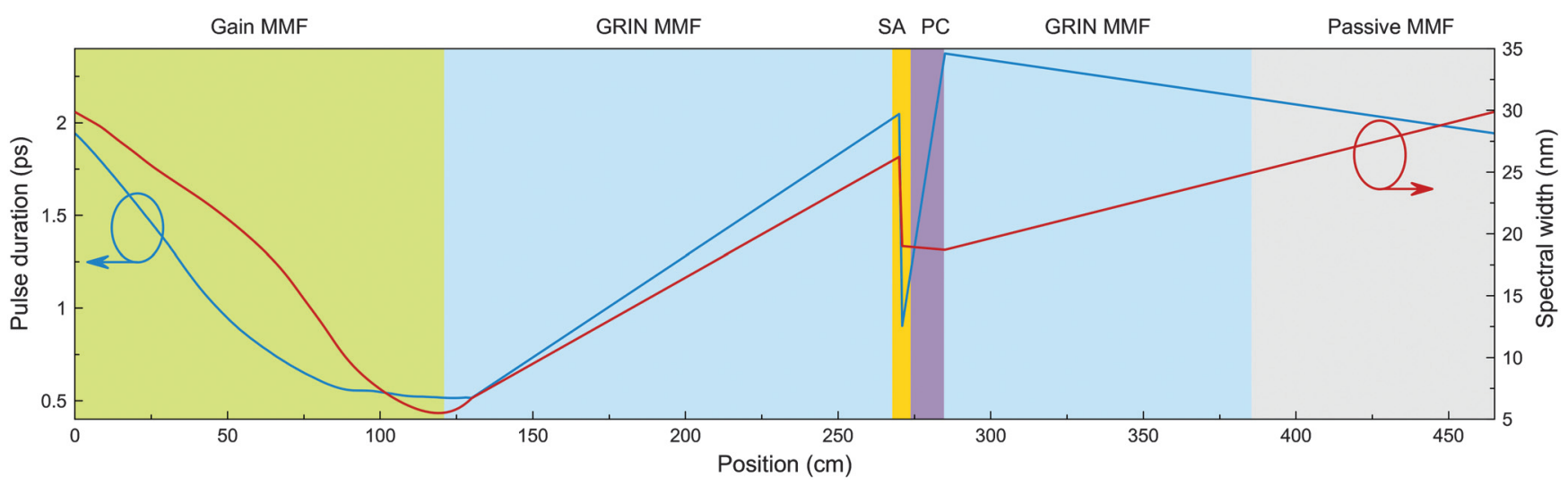

Fig. 2 Pulse evolution in the laser simulations. Pulse duration and spectral bandwidth variation over the cavity. SA, saturable absorber; PC, pulse compressor.
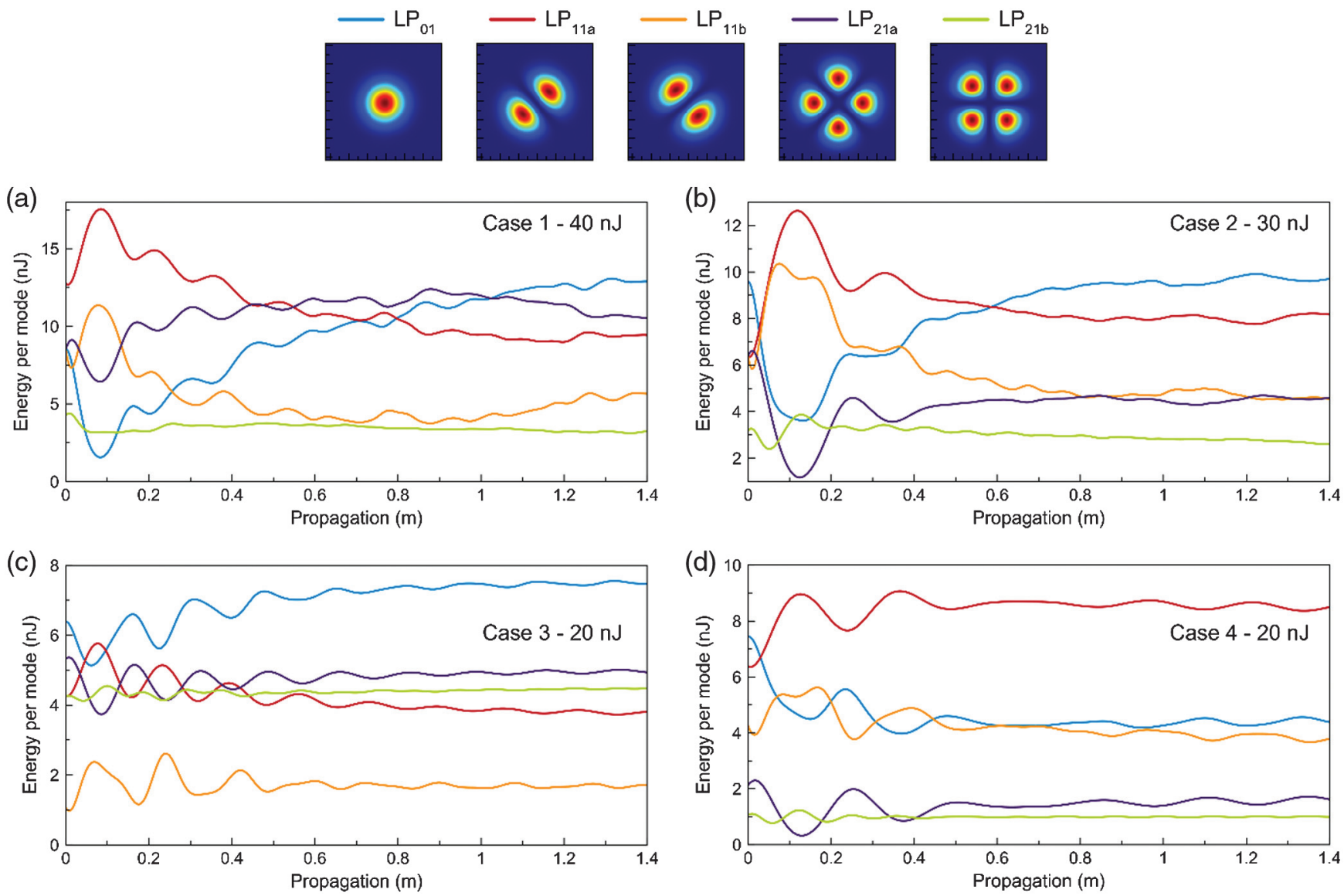

Fig. 3 Numerical investigation of Kerr-induced beam self-cleaning inside GRIN MMF. Propagation of 450 fs pulse with (a) $40 \mathrm{~nJ}$ pulse energy and [20\%, 30\%, 20\%, 20\%, and 10\%] initial coupling condition; (b) $30 \mathrm{~nJ}$ pulse energy and [30\%, 20\%, 20\%, 20\%, and 10\%] initial coupling condition; (c) $20 \mathrm{~nJ}$ pulse energy and [30\%, 20\%, 5\%, 25\%, and 20\%] initial coupling condition; and (d) $20 \mathrm{~nJ}$ pulse energy and [35\%, 30\%, 20\%, 10\%, and 5\%] initial coupling condition.

Contrary to single-pass propagation, inside a laser cavity, a small improvement in beam shape can accumulate, and steady-state beam cleaning can be achieved after multiple roundtrips. The aforementioned numerical studies indicate that the pulse energy required for intracavity Kerr-induced beam cleaning is within reach of the designed dispersion-managed multimode cavity.

\section{Experimental Studies}

Guided by the simulation results, the experimental cavity, presented in Fig. 4(a), is constructed and studied. Here, the utilized fibers are intentionally selected to be identical with the fibers used in the spatiotemporally mode-locked laser literature (GRIN 


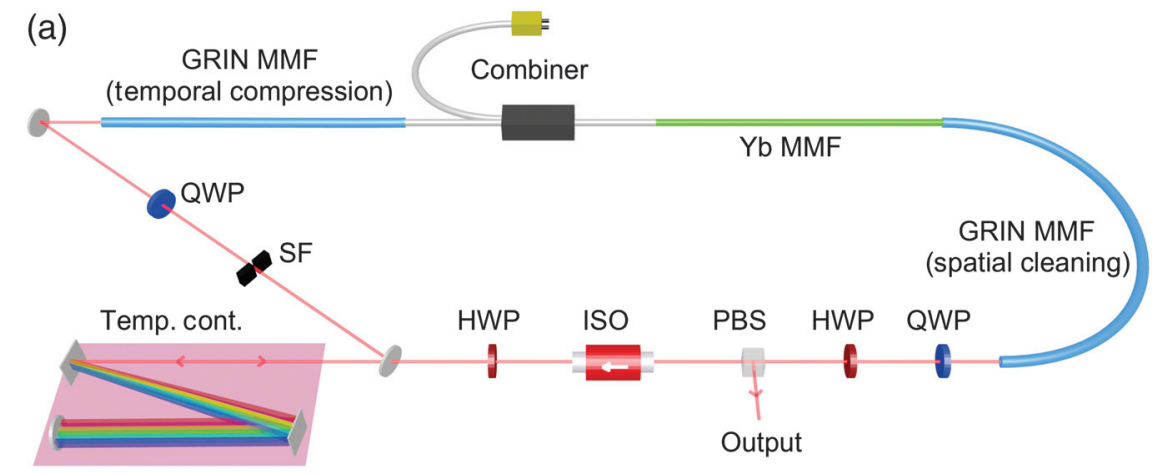

(b)

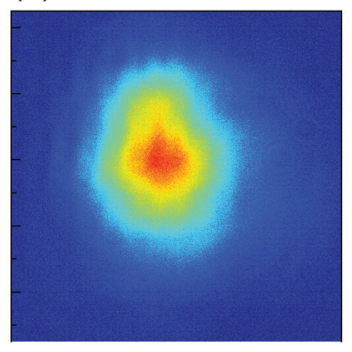

Continuous wave operation

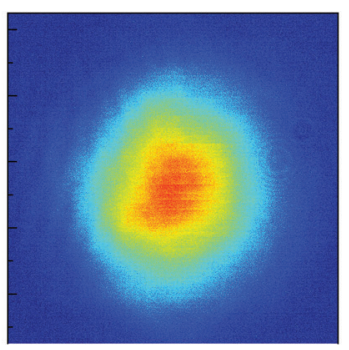

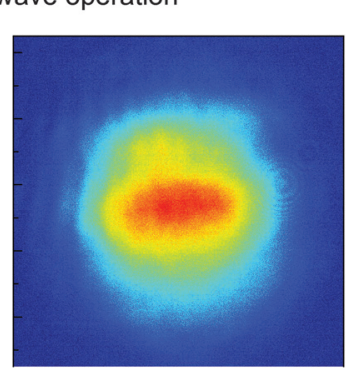

Mode-locked operation
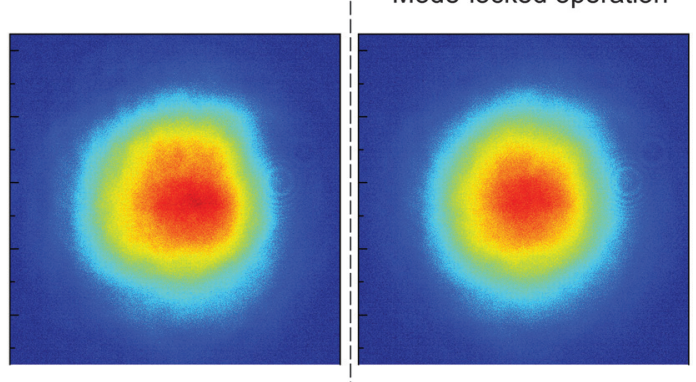

Decreasing cavity loss

Fig. 4 (a) Schematic of the laser cavity and beam profile measurements. QWP, quarter-wave plate; HWP, half-wave plate; PBS, polarizing beam splitter; ISO, isolator; and SF, spatial filter. (b) Evolution of near-field output beam profile for decreasing cavity loss from continuous wave to mode-locked operation for 8-nJ output pulse energy.

MMF with $50 \mu \mathrm{m}$ core diameter and Yb MMF with $10 \mu \mathrm{m}$ core diameter). ${ }^{9-12}$ The GRIN MMF supports 240 modes and Yb MMF supports three modes around $1 \mu \mathrm{m}$ central wavelength. Since the previously reported results in the literature feature highly multimode output beam profiles, instead of the Yb MMF, the GRIN MMF is the dominant waveguide inside the cavity. In addition, offset splice and bending of the GRIN MMF cause multimode propagation to the beam every roundtrip. The offset splice is set to $5 \mu \mathrm{m}$ between the two cores of the fibers. In the literature, Sidelnikov et al. studied the optimized initial conditions for single-pass beam cleaning phenomenon. Their results suggest that precisely a $5 \mu \mathrm{m}$ offset favors nonlinear beam cleaning. ${ }^{20}$ The intracavity grating compressor is tuned to provide $-0.0987 \mathrm{ps}^{2}$ GVD to compensate $0.1116 \mathrm{ps}^{2} \mathrm{GVD}$ of the fiber sections of the cavity each round trip. Single-pulse modelocking is easily achieved by adjusting cavity polarization by the wave plates with a repetition rate of $36 \mathrm{MHz}$. A major improvement from a highly multimode beam profile to a Gaussian-like symmetric beam profile is observed at the laser output when the operation regime changed from continuous-wave to modelocked (see Video S2 [URL: https://doi.org/10.1117/1.AP.2.5 $.056005 .2]$ ). Figure 4(b) presents the multimode nature of the laser cavity for the adjusted cavity loss, and the detailed evolution is presented in Video S3 (URL: https://doi.org/10.1117/ 1.AP.2.5.056005.3). The measured Gaussian-like beam profile remains similar when pulse energy is increased up to $24 \mathrm{~nJ}$ by gradually increasing the pump power level. Above $24 \mathrm{~nJ}$ output pulse energy, secondary pulse formation is observed inside the cavity as a power limiting factor for the single-pulse operation regime.

We performed a detailed characterization of the laser when the pump power is set to $3.5 \mathrm{~W}$, where the laser generates $20 \mathrm{~nJ}$ pulses. As expected for a dispersion-managed cavity, pulse spectra with large bandwidth $(\sim 40 \mathrm{~nm})$ are measured for this power level [see Fig. 5(a)]. The pulse duration of the chirped pulses is measured with second-order intensity autocorrelation as 3.88 ps with the Gaussian deconvolution factor of 1.414 , as shown in Fig. 5(b). These chirped pulses are later dechirped (compressed) by an external grating compressor with a diffraction grating pair to $97 \mathrm{fs}$ [see Fig. 5(c)]. To determine the temporal profile of the pulses from the measured spectrum and autocorrelation data, the PICASO algorithm is employed. ${ }^{21}$ The resulting pulse shape features 95.8 fs pulse duration with a Gaussian profile and is presented in Fig. 5(c) - inset. No secondary pulse formation or periodic oscillation of the pulse train is observed (see Supplementary Discussion II in the Supplementary Material). As presented in Fig. 5(d), the beam has a symmetric shape, and, to determine its quality, $M^{2}$ measurements are performed. For $20 \mathrm{~nJ}$ output pulse energy, the average $M^{2}$ is measured as $1.13\left(M_{x}^{2}=1.08\right.$ and $\left.M_{y}^{2}=1.17\right)$.

\section{Discussion}

To investigate the effect of intracavity pulse energy on output beam profile in detail, we performed $M^{2}$ measurements to pulses with different energies by adjusting the pump power. 

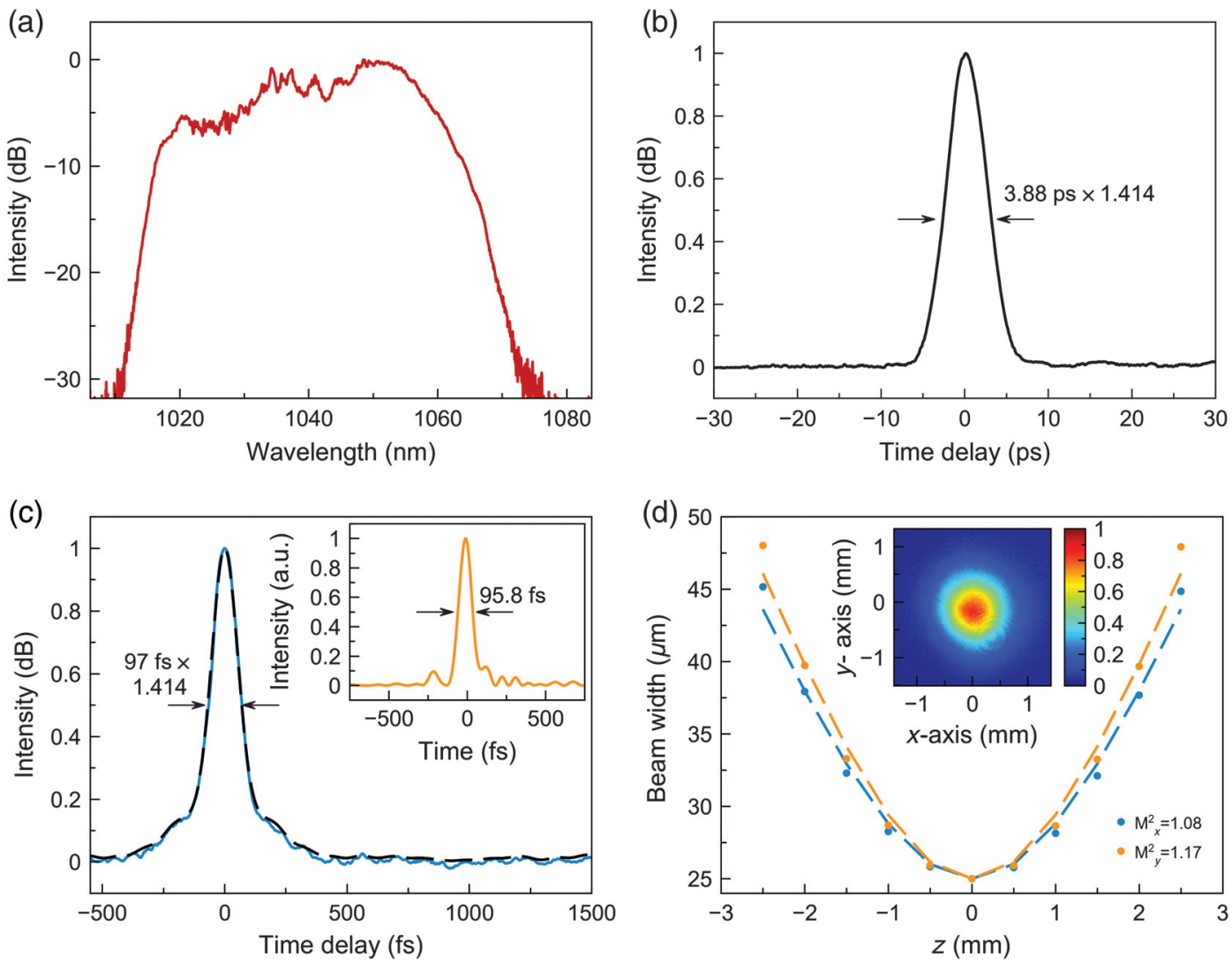

Fig. 5 Experimental measurements: (a) measured mode-locked spectrum; (b) intensity autocorrelation trace of the chirped pulse; (c) intensity autocorrelation trace of the dechirped pulse, PICASO-reconstructed autocorrelation intensity (dashed), and PICASO-retrieved dechirped pulse shape (inset); and (d) measured beam profile for mode-locked operation and $M^{2}$ measurement of the beam profile.

For the spatiotemporally mode-locked beam profile, the changes in the output beam quality are challenging to differentiate from the near-field beam profiles, but $M^{2}$ measurements provide more significant information. In our measurements, we observed that the $M^{2}$ value is decreasing with increasing pulse energy. For $4 \mathrm{~nJ}$ pulses, we measured $M^{2}=1.85$, and it decreased to 1.48 for $7.2 \mathrm{~nJ}$ (Fig. S9 in the Supplementary Material). When the $M^{2}$ value $(<1.13)$ for the presented high pulse energy is also considered, this measurement is the experimental proof of the intracavity Kerr-induced beam cleaning in our design. Here, we would like to emphasize that the observed $M^{2}$ value change from 1.85 to 1.13 is a significant improvement compared to the singlepass beam self-cleaning of ultrashort pulses in the literature, where the $M^{2}$ value improvement is reported only from 2.3 to 1.8 in the initial studies. ${ }^{22}$ Recently, by selecting picosecond pulses at $1.5 \mu \mathrm{m}$ as pump pulses, more efficient beam cleaning results are demonstrated in systems with less modal support. ${ }^{23}$

To understand the beam cleaning mechanism, the effect of the cavity alignment condition on the mode-locked beam profile is investigated. Differences in the free-space orientation of the laser are achieved by changing the cavity alignment and the position of the intracavity spatial filter. We observed that the presented beam-profile remains similar for different alignment configurations, although the mode-locked spectrum and pulse energy change (see Supplementary Discussion II in the Supplementary Material). A comparison of the predicted beam profile by the simulation (Fig. S1c in the Supplementary Material) reveals that experimentally we obtain a much better beam shape [Fig. 5(d)]. One of the reasons for this is the limited number of modes in the simulation and the low-pulse energy that was simulated. In the single-pass simulations reported in Fig. 3, we found that with the higher pulse energy beam cleaning improves, which is consistent with the experimental result. Also, given the fact that the laser pulse oscillates inside the cavity, with a slight improvement in the beam profile due to the Kerr effect during each round trip, enhanced beam self-cleaning is expected.

The difference between the supported numbers of modes by the multimode fibers used in the spatiotemporally mode-locked laser causes mode dependent loss to the propagating field. For spatiotemporal mode-locking with the presented engineered cavity approach, Kerr-induced beam cleaning can cause a minimum loss condition inside the multimode fiber cavity, similar to the Kerr-lens realization of solid-state lasers. Based on our results, one can explain the measured output beam profile evolution in the laser output with the minimum loss principle. ${ }^{24}$ An alternative explanation based on the NPE saturable absorber might also be partly responsible for the experimentally obtained beam cleaning. NPE mode-locking requires nonlinearly induced polarization changes to generate saturable absorber behavior. The low-order modes of the laser cavity exhibit the highest nonlinear rotation angle, thus mode-locking would favor low-order 
modes, which might be another reason for the near single-mode output beam profile achieved in the experiment. However, the majority of the previous sly reported multimode fiber lasers have also employed the nonlinear polarization evolution modelocking technique, and they did not observe a high-quality beam profile. ${ }^{9-11,24}$ Furthermore, we would like to highlight that modification/shaping of the beam in free-space propagation has a very small impact to the output field, since the light travels through an approximately $4 \mathrm{~m}$ coiled multimode fiber with different mode numbers and splice positions. Another important parameter in the demonstrated cavity is the accumulated modal dispersion. Due to the production complexity of gain doped GRIN MMF, we restricted our study of spatiotemporal modelocking studies to commercially available step-index gain MMFs originally designed for amplifier systems. When compared with the step-index MMFs, the modal dispersion of the GRIN MMF segment in the cavity is negligible. For further power scaling by increasing the gain fiber diameter, modal dispersion will be a limiting factor. The aforementioned mechanisms and dynamics will be further investigated in future work. As an interesting future direction, the real-time dynamics of the spatial, temporal, and spectral formation of pulse generation can be investigated numerically and experimentally with DFT-based approaches to understand the mechanism of spatiotemporal mode-locking in the presence of Kerr-induced beam self-cleaning.

In conclusion, we reported a multimode fiber laser design with intracavity Kerr-induced beam self-cleaning to realize high energy, ultrashort pulses with good beam quality. By engineering nonlinear intracavity propagation of the mode-locked pulses, we numerically and experimentally demonstrated a multimode cavity design with Kerr-induced beam self-cleaning. The presented cavity dynamics show that engineered intracavity temporal pulse properties enable a route to generate high beam quality when mode-locking is achieved. For various alignment orientations and pulse energy, drastic improvement of the output beam profile is experimentally reported when mode-locking is achieved. The presented oscillator generates sub-100 fs pulses with $>20 \mathrm{~nJ}$ pulse energy while exhibiting good beam quality if the $M^{2}$ value is $<1.13$. The combination of good beam quality, high pulse energy, and sub-100 fs pulse duration from a fiber laser consisting of commercially available, standardized components is a promising platform for various laser-related fields. The presented technique can be easily adapted to fibers with a larger core size to increase pulse energy while preserving singlepulse operation with sub-100 fs durations.

\section{Acknowledgments}

The authors declare no conflicts of interest.

\section{References}

1. M. E. Fermann and I. Hartl, "Ultrafast fibre lasers," Nat. Photonics 7(11), 868-874 (2013).

2. J. D. Kafka, T. Baer, and D. W. Hall, "Mode-locked erbium-doped fiber laser with soliton pulse shaping," Opt. Lett. 14(22), 12691271 (1989).

3. W. H. Renninger, A. Chong, and F. W. Wise, "Self-similar pulse evolution in an all-normal-dispersion laser," Phys. Rev. A 82(2), 021805 (2010).

4. F. Ö. İlday et al., "Self-similar evolution of parabolic pulses in a laser," Phys. Rev. Lett. 92(21), 213902 (2004).

5. A. Chong et al., "All-normal-dispersion femtosecond fiber laser," Opt. Express 14(21), 10095-10100 (2006).
6. P. Grelu and N. Akhmediev, "Dissipative solitons for mode-locked lasers," Nat. Photonics 6(2), 84-92 (2012).

7. B. Ortaç et al., "Approaching microjoule-level pulse energy with mode-locked femtosecond fiber lasers," Opt. Lett. 34(10), 15851587 (2009).

8. M. Baumgartl et al., "66 W average power from a microjoule-class sub-100 fs fiber oscillator," Opt. Lett. 37(10), 1640-1642 (2012).

9. L. G. Wright, D. N. Christodoulides, and F. W. Wise, "Spatiotemporal mode-locking in multimode fiber lasers," Science 358(6359), 94-97 (2017).

10. H. Qin et al., "Observation of soliton molecules in a spatiotemporal mode-locked multimode fiber laser," Opt. Lett. 43(9), 19821985 (2018).

11. Y. Ding et al., "Multiple-soliton in spatiotemporal mode-locked multimode fiber lasers," Opt. Express 27(8), 11435-11446 (2019).

12. U. Teğin et al., "Spatiotemporal self-similar fiber laser," Optica 6(11), 1412-1415 (2019).

13. K. Krupa et al., "Spatial beam self-cleaning in multimode fibres," Nat. Photonics 11(4), 237-241 (2017).

14. A. Mafi, "Pulse propagation in a short nonlinear graded-index multimode optical fiber," J. Lightwave Technol. 30(17), 28032811 (2012).

15. J. Hult, "A fourth-order Runge-Kutta in the interaction picture method for simulating supercontinuum generation in optical fibers," J. Lightwave Technol. 25(12), 3770-3775 (2007).

16. Y. Chen et al., "Dispersion-managed mode locking," J. Opt. Soc. Am. B 16(11), 1999-2014 (1999).

17. K. Tamura et al., "77-fs pulse generation from a stretched-pulse mode-locked all-fiber ring laser," Opt. Lett. 18(13), 1080-1082 (1993).

18. B. Ortaç et al., "90-fs stretched-pulse ytterbium-doped doubleclad fiber laser," Opt. Lett. 28(15), 1305-1307 (2003).

19. Ç. Şenel et al., "Tailored design of mode-locking dynamics for low-noise frequency-comb generation," Phys. Rev. Appl. 10(2), 024027 (2018).

20. O. S. Sidelnikov et al. "Random mode coupling assists Kerr beam self-cleaning in a graded-index multimode optical fiber," Opt. Fiber Technol. 53, 101994 (2019).

21. J. W. Nicholson et al., "Full-field characterization of femtosecond pulses by spectrum and cross-correlation measurements," Opt. Lett. 24(23), 1774-1776 (1999).

22. Z. Liu et al., "Kerr self-cleaning of femtosecond-pulsed beams in graded-index multimode fiber," Opt. Lett. 41(16), 3675-3678 (2016).

23. Y. Leventoux et al. "Highly efficient few-mode spatial beam selfcleaning at $1.5 \mu \mathrm{m}$," Opt. Express 28(10), 14333-14344 (2020).

24. L. G. Wright et al., "Mechanisms of spatiotemporal modelocking," Nat. Phys. 16(5), 565-570 (2020).

Uğur Teğin received his BSc degree in physics and his MSc degree in material science and nanotechnology from Bilkent University, Ankara, Turkey, in 2015 and 2018, respectively. He is pursuing his PhD in electrical engineering at the École Polytechnique Fédérale de Lausanne (EPFL), Lausanne, Switzerland. His current research interests include fiber lasers and nonlinear fiber optics.

Babak Rahmani received his BSc degree from the Department of Electrical and Computer Engineering, University of Tehran, Tehran, Iran, in 2014 and his MSc degree from Sharif University of Technology, Tehran, Iran, in 2016, both in electrical engineering. Since January 2018, he has been a PhD student at the EPFL, Lausanne, Switzerland. He is interested in computational imaging and application of machine learning for solving inverse problems in photonics.

Eirini Kakkava received her BSc and MSc degrees in physics from the University of Patras, Patras, Greece, in 2013 and 2015, respectively, and her $\mathrm{PhD}$ in photonics from the EPFL, Lausanne, Switzerland, in 2020. Her current research interests include fiber endoscope systems, deep learning, and holography. 
Demetri Psaltis received his BSc, MSc, and $\mathrm{PhD}$ degrees from Carnegie-Mellon University, Pittsburgh, Pennsylvania, USA. He is a professor of optics and the director of the Optics Laboratory at the EPFL, Lausanne, Switzerland. In 1980, he joined the faculty at California Institute of Technology, Pasadena, California, USA. He moved to EPFL in 2006. His research interests include imaging, holography, biophotonics, nonlinear optics, and optofluidics. He has authored or co-authored more than 400 publications in these areas. He is a fellow of the Optical Society of America, the European Optical Society, and SPIE. He was the recipient of the International Commission of Optics Prize, the Humboldt Award, the Leith Medal, and the Gabor Prize.
Christophe Moser received his $\mathrm{PhD}$ from California Institute of Technology in optical information processing in 2000. He is an associate professor of optics and the section director in the Microengineering Department at EPFL. He co-founded and was the CEO of Ondax Inc. (acquired by Coherent Inc.), Monrovia, California, for 10 years before joining EPFL in 2010. His current interests are ultracompact endoscopic optical imaging through multimode fibers, multimode fiber lasers, retinal imaging, and additive manufacturing via volumetric $3 \mathrm{D}$ printing with light. $\mathrm{He}$ is the co-founder of Composyt Light Lab in the field of head-worn displays (acquired by Intel Corp.), Earlysight SA, and Readily3D. He is the author and co-author of 75 peer-reviewed publications and 45 patents. 